\title{
Transverse Momentum, Centrality, and Participant Nucleon Number Dependence of Elliptic Flow
}

\author{
Bao-Chun Li, ${ }^{1}$ Yuan-Yuan Fu, ${ }^{1}$ Li-Li Wang, ${ }^{2}$ and Fu-Hu Liu ${ }^{1}$ \\ ${ }^{1}$ Department of Physics, Shanxi University, Taiyuan, Shanxi 030006, China \\ ${ }^{2}$ Department of Mathematics, Business College of Shanxi University, Taiyuan, Shanxi 030031, China
}

Correspondence should be addressed to Bao-Chun Li; libc2010@163.com

Received 25 July 2012; Revised 9 November 2012; Accepted 12 November 2012

Academic Editor: John W. Norbury

Copyright (C) 2013 Bao-Chun Li et al. This is an open access article distributed under the Creative Commons Attribution License, which permits unrestricted use, distribution, and reproduction in any medium, provided the original work is properly cited.

\begin{abstract}
In the combined framework of a multisource and participant-spectator model, we study the elliptic flow as a function of transverse momentum and number of participating nucleons in $\sqrt{s_{N N}}=200 \mathrm{GeV} \mathrm{Au}-\mathrm{Au}$ and $\sqrt{s_{N N}}=2.76 \mathrm{TeV} \mathrm{Pb}-\mathrm{Pb}$ collisions and argue that the geometry of the initial overlap region in the collisions determines the experimentally measured azimuthal asymmetries. Our results are approximately in agreement with the data from PHENIX and ALICE collaborations.
\end{abstract}

\section{Introduction}

Elliptic flow is one of the key observables to study the properties of the Quark-Gluon plasma (QGP) created at the early stage of high-energy collisions and is generated in a collective expansion of the hot and dense matter. In years of experiments at the relativistic heavy ion collider (RHIC), one of the most important discoveries is the large elliptic flow in noncentral nucleus-nucleus collisions, which is a clear indication of a collective behavior. As a signature of a collective flow in relativistic nuclear collisions, the elliptic flow was also observed at the AGS and SPS. In a thermodynamical picture, the asymmetric distribution of the initial energy density causes a larger pressure gradient in the shortest direction of the ellipsoidal medium, which can turn the spatial anisotropy of the produced hot matter into a flow anisotropy in the momentum distributions of measurable final-state particles $[1,2]$. Therefore, the main interest in the elliptic flow study grew from its sensitivity to the system properties at very early stage in the system evolution.

Elliptic flow is an informative quantity in better understanding the properties of the hot dense matter created during the initial stage of high-energy collisions. Additionally, the study of the elliptic flow dependence on collision geometry provides insight into the degree of thermalization, transport coefficients, and the equation of state (EOS) of the produced medium $[3,4]$. In order to fit the elliptic flow, the hydrodynamic simulation shows that a very small shear viscosity is required $[5,6]$. It is believed that a strongly coupled QGP (sQGP) created at RHIC behaves like a nearly perfect fluid [7]. The ideal hydrodynamics has been successful in explaining the observed collective flow at low transverse momentum [5, 6]. Moreover, many collaborations, such as the STAR Collaboration [8], the PHENIX Collaboration [9], and ALICE Collaboration [10], are involved in this field. For theoretical description or experimental analysis, one can easily find elliptic flows as a function of transverse momentum [9-11], rapidity or pseudorapidity $[12,13]$, centrality percent or impact parameter [14, 15], transverse kinetic energy [11], normalized particle multiplicity [14], and so forth.

The elliptic flow is quantified by the second harmonic Fourier coefficient $v_{2}$ of the azimuthal angle distribution of final-state particles with respect to the reaction plane; that is,

$$
\frac{d N}{d(\phi-\psi)}=N_{0}\left[1+2 v_{2} \cos (2(\phi-\psi))\right]
$$

where $\phi$ is the azimuthal angle, $\psi$ is the event plane angle, and angular brackets denote a statistical average over many particles and events. In our previous work [16], we have investigated the transverse momentum dependence of elliptic 
flow in $\mathrm{Au}-\mathrm{Au}$ collisions at the RHIC energy and in $\mathrm{Pb}$ $\mathrm{Pb}$ collisions at the LHC energy. In this work, we focus our attention on the dependence of elliptic flows on both transverse momentum and number of participants, which is directly related to the centrality and eccentricities in the Glauber model. The elliptic flow dependence on the geometry of collisions is of particular importance, as the flow is thought to depend heavily on the initial spatial anisotropy. Therefore, the basic idea of our model is that the angular azimuthal anisotropy is associated with the geometry of the first stages in the collision, where the projected overlap region is in the shape of an almond with the long axis perpendicular to the interaction plane. This paper is organized as follows. In Section 2, we introduce the model and formulation that builds a connection between the multi-source model $[17,18]$ and the participant-spectator model [19]. In Section 3, we present our results, which are compared with the experimental data. The discussion and the summary are given in Sections 4 and 5.

\section{Elliptic Flow and Number of Participant Nucleons}

In order to understand the elliptic flow dependence on transverse momentum and number of participating nucleons, we use the multi-source model $[17,18]$ and a geometrical picture of the participant-spectator model [19] in this work. By fitting $v_{2}\left(P_{T}\right)$ data, we may find the connection between the two models.

First, we simply describe the multi-source model. At the initial stage of nucleus-nucleus collisions, numerous primary nucleon-nucleon collisions occur. The primary nucleonnucleon collision can be regarded as an emission source at intermediate energy or a few sources at high energy. The participant nucleons in primary collisions have probabilities to take part in cascade collisions. Meanwhile, the particles produced in primary or cascade nucleon-nucleon collisions have probabilities to take part in secondary collisions with the latter nucleons and other particles. Each cascade (or secondary) collision is also regarded as an emission source or a few sources. Many such emission sources of final-state particles are expected to be formed in the collision process, and each source is considered to emit particles isotropically in the rest frame of the source. There are interactions among the emission sources due to the mechanics and electromagnetism effects. The interactions will cause the source to depart from the isotropic emission. In order to describe the anisotropic source, the source deformation and movement have been introduced in the model $[17,18,21]$. In the rest frame of the source, the three momentum components $P_{x}^{\prime}, P_{y}^{\prime}$, and $P_{z}^{\prime}$ of the considered particle have Gaussian distributions with the same standard deviation $\sigma$. The distributions of the momentum components $P_{x}^{\prime}$ and $P_{y}^{\prime}$ are

$$
f_{P_{x, y}^{\prime}}\left(P_{x, y}^{\prime}\right)=\frac{1}{\sqrt{2 \pi} \sigma} \exp \left[-\frac{P_{x, y}^{\prime}}{2 \sigma^{2}}\right] .
$$

Considering the interaction with other emission sources, the source will have deformations and movements along the $o x$ and $o y$ axis; the momentum components will be modified to

$$
P_{x, y}=A_{x, y} P_{x, y}^{\prime}+B_{x, y}
$$

where $A_{x, y}$ and $B_{x, y}$ represent the deformation and movement of the emission source, respectively. Generally speaking, different $A_{x, y}$ or $B_{x, y}$ can be obtained for events with different centralities (or impact parameters). In the ultrarelativistic nuclei collision at the nonzero impact parameter the overlap area in the transverse plane has a short axis (ox) which is parallel to the impact parameter and a long axis which is perpendicular to the impact parameter. Anisotropy in the central region of collisions is driven by the almond shape of the overlapping area $[3,22,23]$, which is converted by the pressure gradient into a momentum asymmetry. More particles are emitted along $o x$ and the anisotropy intensity is denoted by the coefficient $A_{x}$, which is directly sensitive to the change in the initial spatial geometry. As the overlap expands, the system becomes more spherical, and the driving force quenches itself gradually. With the increase of $P_{x}$ or $P_{y}$ of the particles produced in the collisions, the momentum anisotropy decreases. Therefore, for the concerned centrality, $A_{x}$ is not a constant but a function of the momentum. We set empirically the function

$$
A_{x}\left(P_{x}, P_{y}\right)=1+k \exp \left(-\frac{\sqrt{\left(u_{1} P_{x}\right)^{2}+\left(u_{2} P_{y}\right)^{2}}}{\sigma}\right),
$$

where the expansion factor $k$ indicates the magnitude of the expansion and $u_{1}$ and $u_{2}$ denote the contributions of $P_{x}$ and $P_{y}$, respectively. With (3), the joint probability density function of transverse momentum $P_{T}$ and azimuthal angle $\phi$ is

$$
\begin{aligned}
f_{P_{T}, \phi}\left(P_{T}, \phi\right) \\
=P_{T} f_{P_{T}, \phi}\left(P_{T} \cos \phi, P_{T} \sin \phi\right) \\
=\frac{P_{T}}{2 \pi \sigma^{2} A_{x} A_{y}} \\
\quad \times \exp \left[-\frac{\left(P_{T} \cos \phi-B_{x}\right)^{2}}{2 \sigma^{2} A_{x}^{2}}-\frac{\left(P_{T} \sin \phi-B_{y}\right)^{2}}{2 \sigma^{2} A_{y}^{2}}\right] .
\end{aligned}
$$

Then, the elliptic flow is given by

$$
v_{2}=\left\langle\cos \left[2\left(\phi-\psi_{2}\right)\right]\right\rangle,
$$

where $\phi$ is the azimuthal angle of the particle, angular brackets denote a statistical average over many particles and events, and $\psi_{2}$ is an event plane angle and defined as

$$
\psi_{2}=\frac{1}{2} \arctan \frac{\left\langle P_{T} \sin (2 \phi)\right\rangle}{\left\langle P_{T} \cos (2 \phi)\right\rangle} .
$$


For convenience, we can also compute the transverse momentum and elliptic flow by using the Monte Carlo calculation:

$$
\begin{aligned}
& P_{x}=\sigma A_{x} \sqrt{-2 \ln r_{1}} \cos \left(2 \pi r_{2}\right)+B_{x}, \\
& P_{y}=\sigma A_{y} \sqrt{-2 \ln r_{3}} \cos \left(2 \pi r_{4}\right)+B_{y},
\end{aligned}
$$

where $r_{1}, r_{2}, r_{3}$, and $r_{4}$ denote random numbers in $[0,1]$.

The incoming direction of the projectile nucleus is along the $o z$ axis, and the reaction plane is defined as the $x o z$ plane. The minimum distance between the centers of the target and projectile nuclei in collisions is denoted to be the impact parameter $b$. In the fireball participant-spectator model [19], the final particles are emitted from a composite system formed by the overlap between the projectile and target nuclei. According to the participant-spectator model in nucleus-nucleus collisions, the participant is the overlapping region of the two nuclei, and the spectator is the other part outside the overlapping region. Using the geometrical concepts of the participant-spectator model, the nucleon numbers in the target and projectile participants are given by

$$
\begin{aligned}
N_{T}= & \int \rho_{T}\left(\sqrt{x^{2}+y^{2}+z^{2}}\right) \theta\left(R_{T}-\sqrt{x^{2}+y^{2}+z^{2}}\right) \\
& \times \theta\left[R_{P}-\sqrt{(x-b)^{2}+y^{2}}\right] d x d y d z, \\
N_{P}= & \int \rho_{P}\left[\sqrt{(x-b)^{2}+y^{2}+z^{2}}\right] \\
& \times \theta\left[R_{P}-\sqrt{(x-b)^{2}+y^{2}+z^{2}}\right] \\
& \times \theta\left(R_{T}-\sqrt{x^{2}+y^{2}}\right) d x d y d z,
\end{aligned}
$$

respectively, where $R_{P}$ and $R_{T}$ are the projectile and target radius. The nuclear density function for an even distribution is $\rho=3 / 4 \pi r_{0}^{3}$, and for a heavy nucleus with mass number greater than 16, there is a two-parameter Fermi distribution [24]. Then, we have

$$
\begin{gathered}
\rho_{T}\left(\sqrt{x^{2}+y^{2}+z^{2}}\right)=\frac{\rho_{0}}{1+\exp \left[\left(\sqrt{x^{2}+y^{2}+z^{2}}-R_{T}\right) / \alpha\right]}, \\
\rho_{P}\left[\sqrt{(x-b)^{2}+y^{2}+z^{2}}\right] \\
=\frac{\rho_{0}}{1+\exp \left\{\left[\sqrt{(x-b)^{2}+y^{2}+z^{2}}-R_{P}\right] / \alpha\right\}},
\end{gathered}
$$

respectively, where $\rho_{0}, R_{T}\left(R_{P}\right)$, and $\alpha$ represent the normalized constant, nuclear radius, and skin depth, respectively. The parameters are taken as $R_{T}=R_{P}=R_{\mathrm{Au}}=6.38 \mathrm{fm}$ and $\alpha=0.535 \mathrm{fm}$, for ${ }^{197} \mathrm{Au}+{ }^{197} \mathrm{Au}$, and $R_{T}=R_{P}=R_{\mathrm{Pb}}=$ $6.62 \mathrm{fm}$, and $\alpha=0.546 \mathrm{fm}$, for ${ }^{208} \mathrm{~Pb}+{ }^{208} \mathrm{~Pb}$, based on data from low energy electron-nucleus scattering experiments [25]. Although the nuclear densities of $\mathrm{Au}$ and $\mathrm{Pb}$ nuclei are the experimental charge densities, the difference in the neutron densities has little effect on the number of participant nucleons. The total nucleon numbers in the two participants are

$$
N_{\text {part }}=N_{P}+N_{T}
$$

As can be clearly seen from the above discussion, the correlation between $b$ and $N_{\text {part }}$ calculated by the geometrical structure named the participant-spectator model has been given. $N_{\text {part }}$ is a well-understood quantity that is directly related to the centrality and calculated eccentricities in the Glauber model. However, we also want to know the relationship between the elliptic flow and the number of participants. So, it is naturally that the multi-source model was introduced from previous works.

\section{Comparison with Experimental Data}

In Figure 1, we show elliptic flows $v_{2}$ as a function of transverse momentum $P_{T}$ for charged hadrons produced in $\sqrt{s_{N N}}=200 \mathrm{GeV}$ Au-Au collisions and $\sqrt{s_{N N}}=2.76 \mathrm{TeV}$ $\mathrm{Pb}-\mathrm{Pb}$ collisions. The symbols represent the experimental data of the PHENIX Collaboration [20] and the ALICE Collaboration [10]. The curves are our computed results with different $k$, which are determined by fitting the data and are given as a function of centrality percent $c$ in Figure 2(a). The other parameters used for the calculations are $u_{1}=0.94$ and $u_{2}=0.59-0.61$ (Figure $1(\mathrm{a})$ ) and $u_{1}=0.988$ and $u_{2}=0.625-0.640$ (Figure 1(b)), which are determined by fitting the data and the increase with center of energy and are independent of centralities. It is obvious that $v_{2}$ increases with $P_{T}$ in the low $P_{T}$ region, as predicted by ideal hydrodynamic calculations. The observed $v_{2}$ saturates or decreases in the region of $P_{T}>2 \mathrm{GeV} / c$. However, ideal hydrodynamic model calculations show that $v_{2}$ increases with $P_{T}$ in this region [4]. One can see that the calculated results are in agreement with the experimental data in the whole observed $P_{T}$ region for all concerned centralities.

The square values of the expansion factor $k^{2}$ used for Figure 1 are displayed in Figure 2(a), by different symbols as marked. Obviously, $v_{2}\left(P_{T}\right)$ tends to a saturation for the more peripheral collisions $(>50 \%)$. We find that, except for the saturation region, the values of $k^{2}$ are approximately proportional to the centrality percent $c$. By the geometric relation of the $c$ to the impact parameter $b$ [27], we have

$$
c \simeq \frac{b^{2}}{\left(2 R_{\mathrm{Au}(\mathrm{Pb})}\right)^{2}},
$$

which holds to a very high accuracy for all but most peripheral collisions, and $R_{\mathrm{Au}(\mathrm{Pb})}$ represents the nuclear radius of $\mathrm{Au}(\mathrm{Pb})$. Then, the dependence of the expansion factor $k$ on the $b$ is obtained and displayed in Figure 2(b). $k$ increases linearly with increasing the impact parameter $b$ from the most central $(0-10 \%)$ to the mid-peripheral (40$50 \%)$ collisions. From Figure 1, we notice that $v_{2}\left(P_{T}\right)$ tends to saturation for the more peripheral collisions $(>50 \%)$ in $\sqrt{s_{N N}}=200 \mathrm{GeV}$ Au-Au collisions and $\sqrt{s_{N N}}=2.76 \mathrm{TeV} \mathrm{Pb}-$ $\mathrm{Pb}$ collisions. So, it renders that $k$ has a saturation at large $b$. 

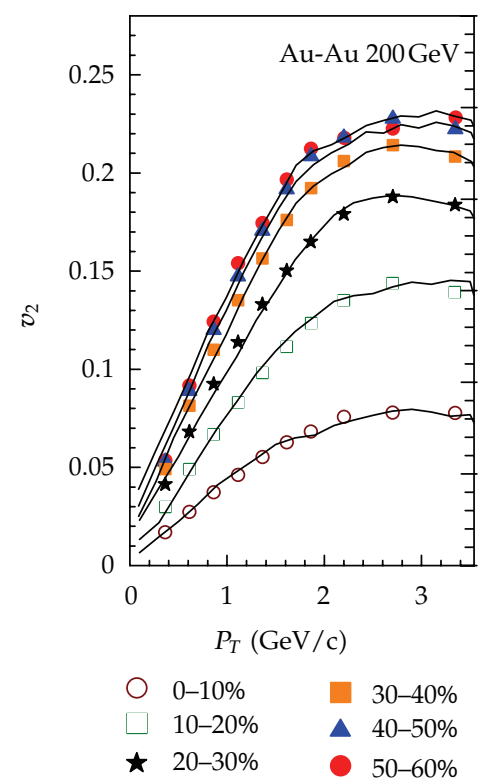

(a)

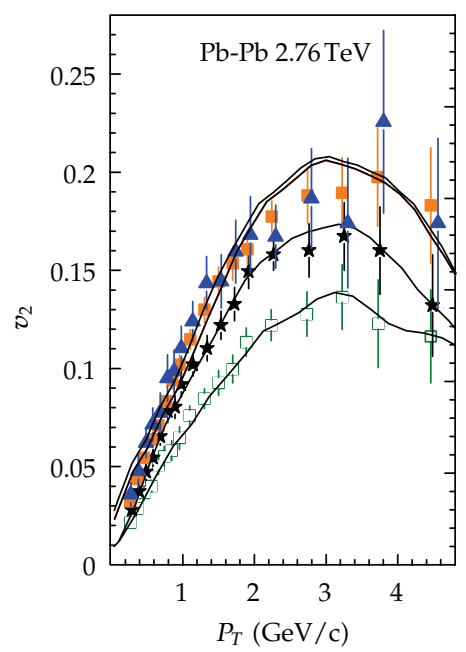

(b)

FIgURE 1: $v_{2}$ versus $P_{T}$ in $\sqrt{s_{N N}}=200 \mathrm{GeV}$ Au-Au collisions and $\sqrt{s_{N N}}=2.76 \mathrm{TeV} \mathrm{Pb}-\mathrm{Pb}$ collisions. Experimental data taken from the PHENIX Collaboration [20] and the ALICE Collaboration [10] are shown with the scattered symbols. Our results calculated from the multisource model are shown with the curves.

As shown in Figure 2(b), the saturation happens at $b>8.0 \mathrm{fm}$. Except for the saturation region, the values of $k$ exhibit a linear dependence on the impact parameter $b$. This linear dependence corresponds to a range from the most central $(0-10 \%)$ to the mid-peripheral $(40-50 \%)$ collisions. For Au$\mathrm{Au}$ and $\mathrm{Pb}-\mathrm{Pb}$ collisions, in the linear (or non-saturation) region, we obtain $k=(0.124 \pm 0.045) b+(0.031 \pm 0.004)$ and $k=(0.137 \pm 0.034) b+(0.048 \pm 0.005)$, respectively. The slope for $\mathrm{Pb}-\mathrm{Pb}$ collisions increases by about $10 \%$ over $\mathrm{Au}-\mathrm{Au}$ collisions.

In heavy-ion collisions, initial geometric quantities such as the impact parameter and the shape of the collision

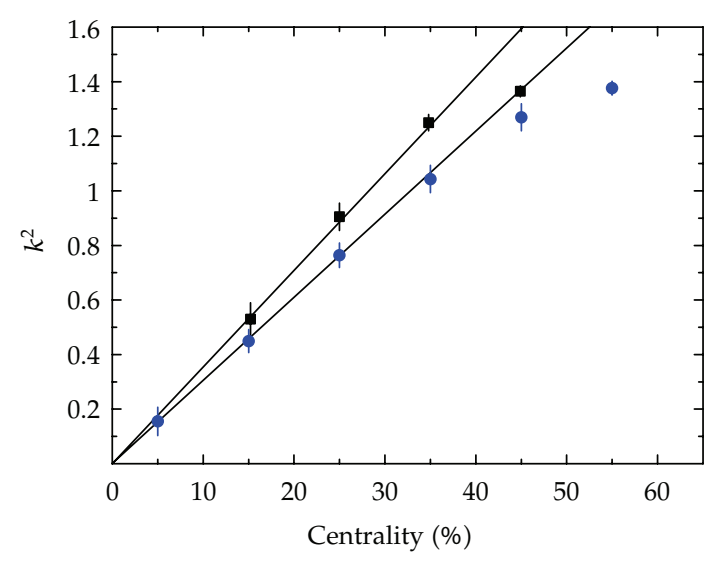

- $\mathrm{Pb}-\mathrm{Pb} 2.76 \mathrm{TeV}$

- Au-Au $200 \mathrm{GeV}$

(a)

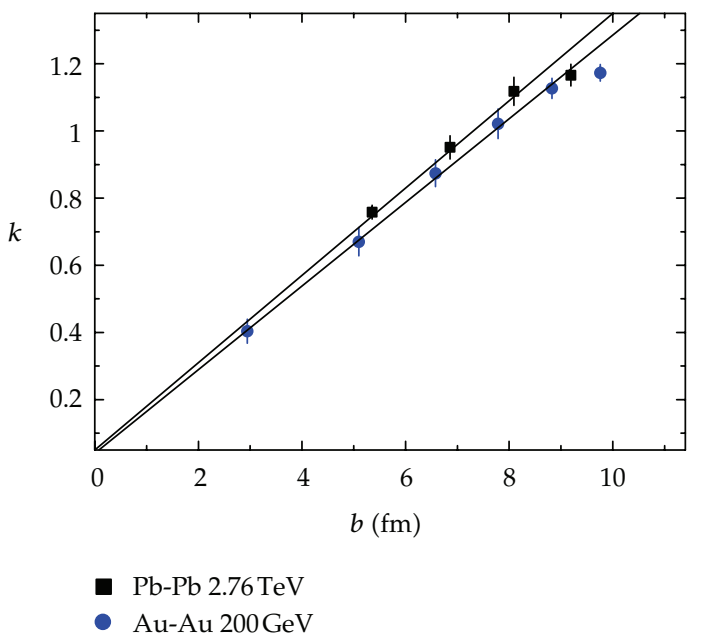

(b)

FIGURE 2: The dependence of the expansion factor $k$ on the centrality and the impactor parameter $b$. The symbols represent the parameter values used in Figure 1. The lines are fitted results.

region cannot be directly determined experimentally. However, it is possible to relate the elliptic flow and number of observed particles to the centrality of the collisions. Using the percentile centrality of a collision, the initial geometric configuration can be estimated with models [23, 27-30]. In terms of elliptic flows, the almond shaped interaction volume produced in a noncentral collision is converted by the pressure gradient into a momentum asymmetry. For final particles, the interaction of the emission sources is related to the hot dense matter in sources and also results in the azimuthally anisotropic expansion in the momentum space [31].

With fitting the data of $v_{2}\left(P_{T}\right)$, the parameters used for the calculations are obtained. And then, combining the above linear relation between $k$ and $b$ with (12), we can compute the elliptic flows $v_{2}$ as a function of the participant nucleon number $N_{\text {part }}$ in $\sqrt{s_{N N}}=200 \mathrm{GeV}$ Au-Au collisions and $\sqrt{s_{N N}}=2.76 \mathrm{TeV} \mathrm{Pb}-\mathrm{Pb}$ collisions, which are shown in 


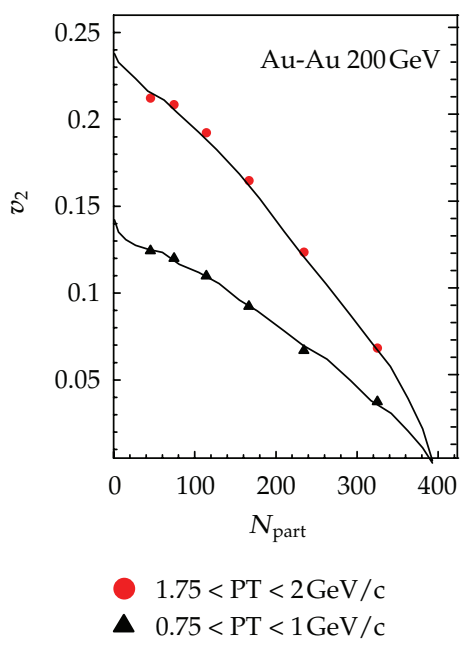

(a)

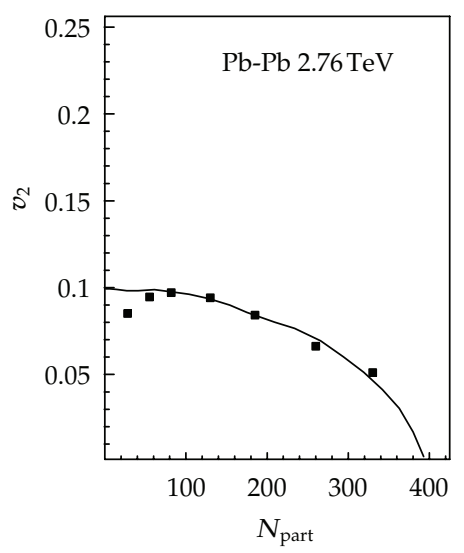

(b)

Figure 3: Dependences of $v_{2}$ on $N_{\text {part }}$ in $\sqrt{s_{N N}}=200 \mathrm{GeV} \mathrm{Au-}$ $\mathrm{Au}$ collisions and $\sqrt{s_{N N}}=2.76 \mathrm{TeV} \mathrm{Pb}-\mathrm{Pb}$ collisions. Experimental data taken from the PHENIX Collaboration [20] and the ALICE Collaboration [26] are shown with the scattered symbols. The curves are our calculated results. The error bars are smaller than the size of the data point.

Figures 3(a) and 3(b), respectively. The symbols represent the experimental data of the PHENIX Collaboration [20] and the ALICE Collaboration [26]. It is obvious that $v_{2}$ decreases with the increase of $N_{\text {part }}$. One can see that except for the very peripheral collisions (very low $N_{\text {part }}$ ), the calculated results are approximately in agreement with the experimental data of $N_{\text {part }}$ for different transverse momentum $P_{T}$ ranges.

\section{Discussion of the Results}

We have presented the results of azimuthal anisotropy using the elliptic flow $v_{2}$ as a function of $P_{T}$ and $N_{\text {part }}$ in $\sqrt{s_{N N}}=$ $200 \mathrm{GeV} \mathrm{Au}-\mathrm{Au}$ collisions and $\sqrt{s_{N N}}=2.76 \mathrm{TeV} \mathrm{Pb}-\mathrm{Pb}$ collisions. Elliptic flow dependence on $P_{T}$ is well described for a wide range of transverse momentum values in the framework of the multi-source model. The parameter $A_{x}$ in the model is used to reflect the expansion of momenta. An isotropic emission corresponds to the expansion factor $k=0$. Significantly, the detailed comparison between the calculation results and the experimental data proved that $k$ increases linearly with the impact parameter $b$ from the most central $(0-10 \%)$ to mid-peripheral (40-50\%) collisions. The behavior is due to the change in initial eccentricity of the initial fireball from central to peripheral events at each centrality $[32,33]$. In the combined analysis of the multi-source model and the participant-spectator model, the double differential flow coefficients $v_{2}\left(P_{T}, N_{\text {part }}\right)$ can be obtained. It is found that except for the very peripheral collisions, the results are approximately in agreement with the experimental data from the PHENIX and ALICE Collaborations.

Our treatment of $v_{2}-N_{\text {part }}$ correlation is very simple. According to the participant-spectator model [23], the participant nucleon number $N_{\text {part }}$ at a given impact parameter $b$ can be calculated by a geometrical picture. $N_{\text {part }}$ is a wellunderstood quantity that is directly related to the centrality and the calculated eccentricities in the Glauber model. The expansion coefficient $k$ in the multi-source model $[17,18]$ is related to $b$ (or centrality percent $c$ ) by fitting the data of $v_{2}\left(P_{T}\right)$, where a linear relationship between $k$ and $b$ is obtained by using a realistic description of the nuclear geometry in a Glauber calculation [24]. In this case, a connection between $v_{2}\left(P_{T}\right)$ and $N_{\text {part }}$ via $b$ has been built in the combined framework of the two models. Such geometric calculations will be helpful to understand the baseline physics of heavy ions at the LHC in terms of nuclear geometry. Future heavy ion experiments, both at the RHIC and the LHC, will further push our understanding of nuclear geometry.

The elliptic flow originates from the spatial anisotropy of the initial overlap volume in noncentral nucleus-nucleus collisions. It is one of the most informative quantities in better understanding the nature and properties of the matter in high-energy nuclear collisions. The ideal hydrodynamic model calculations reproduce the mass ordering of $v_{2}$ in the relatively low $P_{T}$ region but overshoot the values of $v_{2}$ for all centrality bins [34]. To understand the viscous nature of QGP, the dissipative hydrodynamics have recently been applied to explain the experimental data of $v_{2}$ by including the effect of shear and bulk viscosity [35-37]. The study of the elliptic flow in our simple model, where the system expansion can be quantified in the momentum space, shows that the expansion factor $k$ is expressed by the impact parameter $b$, which is related to the number of participants $N_{\text {part }}$ using a realistic description of the nuclear geometry in a Glauber calculation [24].

\section{Summary and Conclusions}

Summarizing up, using the combined framework of the multi-source model $[17,18]$ and the participant-spectator model [19], we have investigated the elliptic flow of final-state particles produced in nucleus-nucleus collisions. The model is able to describe the dependence of the elliptical flow on transverse momentum and number of participants (or centrality). The model is too simplistic to give the geometrical picture of nucleus-nucleus collisions. However, it proves to be helpful 
to understand the anisotropic momentum distribution and $N_{\text {part }}$ (or centrality) dependence of various elliptic flows. Particularly, this model is successful in the descriptions of (pseudo) rapidity and multiplicity distributions for produced particles $[17,18]$. In the description of $v_{2}\left(P_{T}, N_{\text {part }}\right)$, the present work is a successful attempt.

\section{Acknowledgments}

This work is supported by the National Natural Science Foundation of China under Grant no. 11247250, no. 10975095 and no. 11005071, the National Fundamental Fund of Personnel Training (J1103210), the Shanxi Provincial Natural Science Foundation under Grant no. 2011011001, and the Open Research Subject of the Chinese Academy of SciencesLarge-Scale Scientific Facility (2060205).

\section{References}

[1] V. P. Konchakovski, E. L. Bratkovskaya, W. Cassing, V. D. Toneev, S. A. Voloshin, and V. Voronyuk, "Azimuthal anisotropies for $\mathrm{Au}+\mathrm{Au}$ collisions in the parton-hadron transient energy range," Physical Review C, vol. 85, Article ID 044922, 2012.

[2] M. Petrovici and A. Pop, "Collective phenomena in heavy ion collisions," Romanian Journal of Physics, vol. 57, pp. 419-431, 2012.

[3] H. J. Drescher, A. Dumitru, C. Gombeaud, and J. Y. Ollitrault, "Centrality dependence of elliptic flow, the hydrodynamic limit, and the viscosity of hot QCD," Physical Review C, vol. 76, no. 2, Article ID 024905, 2007.

[4] P. Huovinen and P. V. Ruuskanen, "Hydrodynamic models for heavy ion collisions," Annual Review of Nuclear and Particle Science, vol. 56, pp. 163-206, 2006.

[5] D. Teaney, J. Lauret, and E. V. Shuryak, "Flow at the SPS and RHIC as a Quark-Gluon plasma signature," Physical Review Letters, vol. 86, pp. 4783-4786, 2001.

[6] P. Huovinen, P. F. Kolb, U. W. Heinz, P. V. Ruuskanen, and S. A. Voloshin, "Radial and elliptic flow at RHIC: further predictions," Physics Letters B, vol. 503, pp. 58-64, 2001.

[7] H. Song and U. W. Heinz, "Suppression of elliptic flow in a minimally viscous quark-gluon plasma," Physics Letters $B$, vol. 658, p. 279, 2008.

[8] C. Adler, Z. Ahammed, C. Allgower et al., "Elliptic flow from two- and four-particle correlations in $\mathrm{Au}+\mathrm{Au}$ collisions at $\sqrt{s_{N N}}=130$ GeV," Physical Review C, vol. 66, Article ID 034904 , 2002 .

[9] S. Afanasiev et al., "Elliptic flow for $\phi$ mesons and (Anti)deuterons in Au+Au collisions at $\sqrt{s_{N N}}=200 \mathrm{GeV}$," Physical Review Letters, vol. 99, Article ID 052301, 2007.

[10] K. Aamodt, B. Abelev, A. Abrahantes et al., "Elliptic ow of charged particles in $\mathrm{Pb}-\mathrm{Pb}$ collisions at $\sqrt{s_{N N}}=2.76 \mathrm{TeV}$," Physical Review Letters, vol. 105, Article ID 252302, 2010.

[11] Y. Oh, Z. W. Lin, and C. M. Ko, "Deuteron production and elliptic flow in relativistic heavy ion collisions," Physical Review C, vol. 80, Article ID 064902, 2009.

[12] B. Yu. Ivanov, I. N. Mishustin, V. N. Russkikh, and L. M. Satarov, "Elliptic flow and dissipation in heavy-ion collisions at $E_{\text {lab }} \simeq$ (1 - 160)A GeV," Physical Review C, vol. 80, Article ID 064904, 2009.
[13] M. Nasim, L. Kumar, P. K. Netrakanti, and B. Mohanty, "Energy dependence of elliptic flow from heavy-ion collision models," Physical Review C, vol. 82, Article ID 054908, 2010.

[14] D. d'Enterria, G. K. Eyyubova, V. L. Korotkikh et al., "Estimates of hadron azimuthal anisotropy from multiparton interactions in proton-proton collisions at $\sqrt{s}=14 \mathrm{TeV}$,' European Physical Journal C, vol. 66, no. 1, pp. 173-185, 2010.

[15] M. Luzum, "Elliptic flow at energies available at the CERN Large Hadron Collider: comparing heavy-ion data to viscous hydrodynamic predictions," Physical Review C, vol. 83, Article ID 044911, 2011.

[16] B.-C. Li, Y.-Y. Fu, L.-L. Wang, E.-Q. Wang, X.-J. Wen, and F.$\mathrm{H}$. Liu, "Dependence of elliptic flow on transverse momentum in $\sqrt{s_{N N}}=200 \mathrm{GeV} \mathrm{Au}-\mathrm{Au}$ and $\sqrt{s_{N N}}=2.76 \mathrm{TeV} \mathrm{Pb}-\mathrm{Pb}$ collisions," Chinese Physics Letters, vol. 29, Article ID 072501, 2012.

[17] F. H. Liu, J. S. Li, and M. Y. Duan, "Light fragment emission in ${ }^{86} \mathrm{Kr}^{124} \mathrm{Sn}$ collisions at $25 \mathrm{MeV} /$ nucleon," Physical Review $\mathrm{C}$, vol. 75, Article ID 054613, 2007.

[18] J. X. Sun, F. H. Liu, E. Q. Wang, Y. Sun, and Z. Sun, "Pseudorapidity distributions of charged particles in $p-\bar{p}$ or $\mathrm{p}-\mathrm{p}$ collisions at high energies and predictions at ultrahigh energies," Physical Review C, vol. 83, Article ID 014001, 2011.

[19] J. Gosset, H. H. Gutbrod, W. G. Meyer et al., "Central collisions of relativistic heavy ions," Physical Review C, vol. 16, pp. 629657, 1977.

[20] A. Adare, S. Afanasiev, C. Aidala et al., "Measurements of higher order flow harmonics in Au+Au collisions at $\sqrt{s_{N N}}=200 \mathrm{GeV}$," Physical Review Letters, vol. 107, Article ID 252301, 2011.

[21] B.-C. Li, Y.-Y. Fu, L.-L. Wang, E.-Q. Wang, and F.-H. Liu, "Transverse momentum distributions of strange hadrons produced in nucleus-nucleus collisions at $\sqrt{s_{N N}}=62.4$ and 200 GeV," Journal of Physics G, vol. 39, Article ID 025009, 2012.

[22] H. Sorge, "Elliptical flow: a signature for early pressure in ultrarelativistic nucleus-nucleus collisions," Physical Review Letters, vol. 78, pp. 2309-2312, 1997.

[23] R. Snellings, "Elliptic flow: a brief review," New Journal of Physics, vol. 13, Article ID 055008, 2011.

[24] M. L. Miller, K. Reygers, S. J. Sanders, and P. Steinberg, "Glauber modeling in high-energy nuclear collisions," Annual Review of Nuclear and Particle Science, vol. 57, p. 205, 2007.

[25] H. De Vries, C. W. De Jager, and C. De Vries, "Nuclear charge-density-distribution parameters from elastic electron scattering," Atomic Data and Nuclear Data Tables, vol. 36, no. 3, pp. 495-536, 1987.

[26] K. Aamodt, B. Abelev, A. Abrahantes Quintana et al., "Higher harmonic anisotropic flow measurements of charged particles in $\mathrm{Pb}-\mathrm{Pb}$ collisions at $\sqrt{s_{N N}}=2.76 \mathrm{TeV}$," Physical Review Letters, vol. 107, Article ID 032301, 2011.

[27] W. Broniowski and W. Florkowski, "Geometric relation between centrality and the impact parameter in relativistic heavy-ion collisions," Physical Review C, vol. 65, Article ID 024905, 2002.

[28] H. Appelshauser et al., "Spectator nucleons in $\mathrm{Pb}+\mathrm{Pb}$ collisions at $158 \mathrm{~A} \cdot \mathrm{GeV}$," European Physical Journal A, vol. 2, pp. 383-390, 1998.

[29] B. Alver, M. Baker, C. Loizides, and P. Steinberg, "The PHOBOS Glauber Monte Carlo," http://arxiv.org/abs/arXiv:0805.4411.

[30] A. D. Sood and R. K. Puri, "The study of participant-spectator matter and collision dynamics in heavy-ion collisions," International Journal of Modern Physics E, vol. 15, p. 899, 2006. 
[31] T. Song, C. M. Ko, S. H. Lee, and J. Xu, "J/ $\psi$ production and elliptic flow in relativistic heavy-ion collisions," Physical Review C, vol. 83, Article ID 014914, 2011.

[32] R. S. Bhalerao, J. P. Blaizot, N. Borghini, and J. Y. Ollitrault, "Elliptic flow and incomplete equilibration at RHIC," Physics Letters B, vol. 627, no. 1-4, pp. 49-54, 2005.

[33] W. Broniowski, P. Bozek, and M. Rybczynski, "Fluctuating initial conditions in heavy ion collisions from the Glauber approach," Physical Review C, vol. 76, Article ID 054905, 2007.

[34] B. I. Abelev, M. M. Aggarwal, Z. Ahammed et al., "Charged and strange hadron elliptic flow in $\mathrm{Cu}+\mathrm{Cu}$ collisions at $\sqrt{s_{N N}}=62.4$ and 200 GeV," Physical Review C, vol. 81, Article ID 044902, 2010.

[35] G. S. Denicol, T. Kodama, and T. Koide, "The effect of shear and bulk viscosities on elliptic flow," Journal of Physics G, vol. 37, Article ID 094040, 2010, http://arxiv.org/abs/1002.2394.

[36] B. Schenke, S. Jeon, and C. Gale, "Anisotropic flow in sqrt(s)= 2.76 TeV $\mathrm{Pb}+\mathrm{Pb}$ collisions at the LHC," Physics Letters B, vol. 702, pp. 59-63, 2011, http://arxiv.org/abs/1102.0575.

[37] C. Shen and U. Heinz, "Hydrodynamic flow in heavy-ion collisions with large hadronic viscosity," Physical Review C, vol. 83, Article ID 044909, 2011, http://arxiv.org/abs/1101.3703/. 

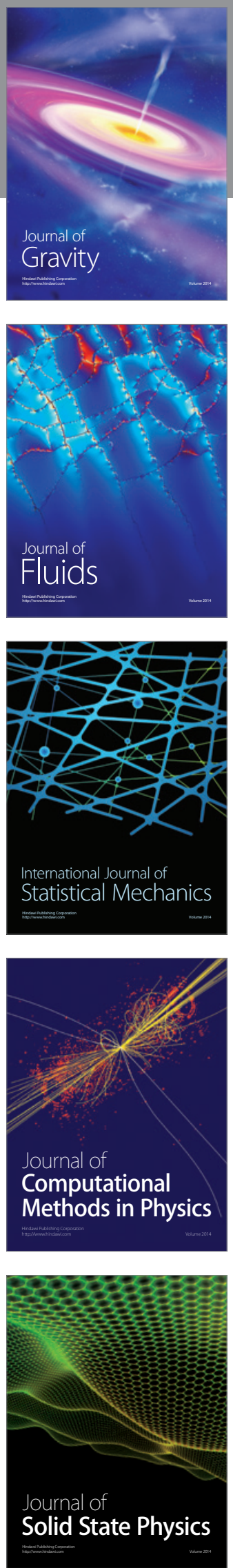

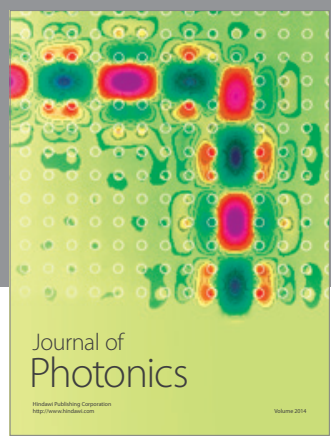

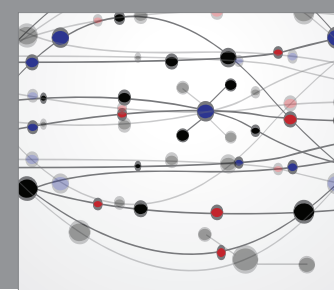

The Scientific World Journal

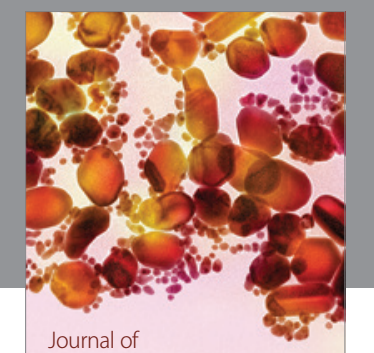

Soft Matter
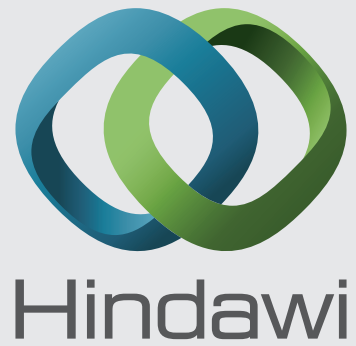

Submit your manuscripts at

http://www.hindawi.com
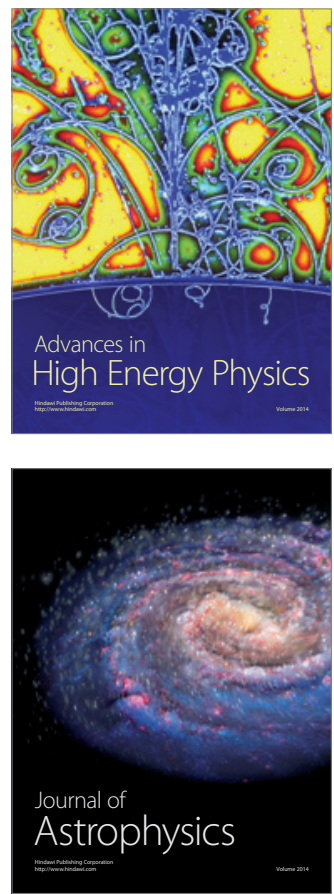
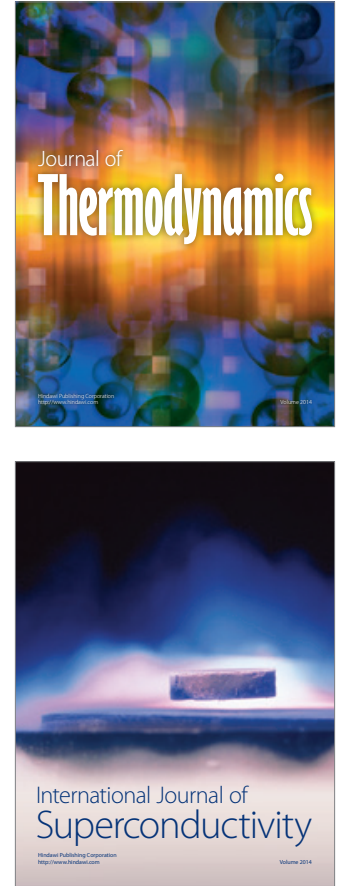
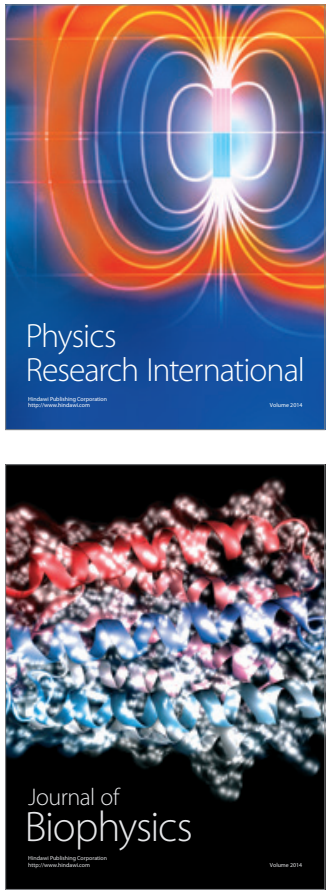
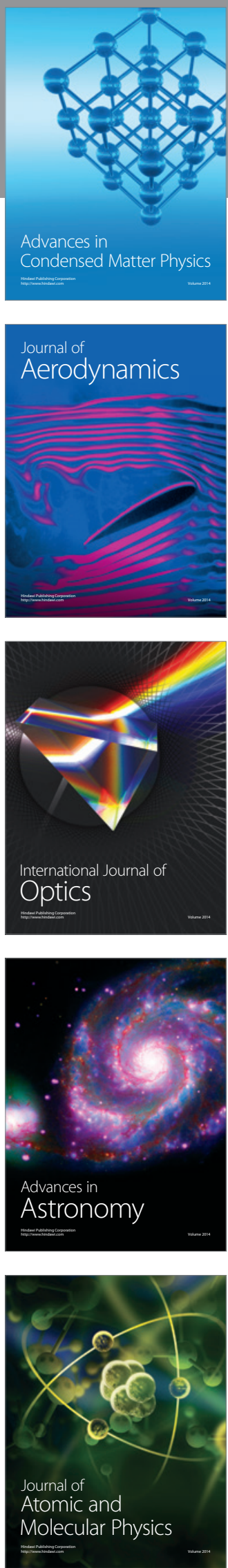